\title{
The Quantum Mechanism of AGNs' Jets
}

\author{
Amrit S. Šorli ${ }^{1,2}$ \\ ${ }^{1}$ Bijective Physics Institute, Slovenia \\ ${ }^{2}$ Scientific research centre Bistra, Slovenija \\ sorli.bijective.physics@gmail.com \\ https://orcid.org/0000-0001-6711-4844 \\ Štefan Čelan \\ Scientific research centre Bistra, Slovenija \\ stefan.celan@bistra.si \\ https://orcid.org/0000-0003-3646-1469
}

\begin{abstract}
Active galactic nuclei (AGNs) are throwing in the interstellar space huge jets of energy in the form of elementary particles. The calculation of the energy density of space in the centre of the black hole with the mass of the Sun shows that in the space-time singularity of such a black hole energy density of space there is so low that atoms become unstable and fall apart into elementary particles. In this sense, AGN is a rejuvenating system of the universe. It transforms its own old matter into fresh energy in the form of jets.
\end{abstract}

Keywords: Space-time singularities; Energy density of quantum vacuum; AGN; Jets.

\section{Introduction}

Several pieces of research suggest that superfluid quantum vacuum also named superfluid quantum space is the physical origin of the universal space $[1,2,3,4]$. The idea of space-time as the fundamental arena of the universe is replaced by the idea that universal space is a type of energy that has superfluid properties. One of these superfluid properties is that every physical object is diminishing the Planck energy density $\rho_{\mathrm{EP}}$ of the superfluid quantum vacuum which is the origin of the universal space in its centre exactly for the amount of its mass $m$ and energy E:

$$
E=m c^{2}=\left(\rho_{E P}-\rho_{E c}\right) V
$$

where $\rho_{\min }$ is the energy density of the universal space in the centre of the physical object and $V$ is the volume of the object [3]. The "no hair theorem" states that a black hole can be defined by three parameters: mass, electric charge, angular momentum [5]. Considering the variable 
energy density of universal space, I introduce a new parameter, the "minimal energy density of space in the centre of a black hole". By Eq. (1), we get:

$$
\rho_{E c}=\rho_{E P}-\frac{m c^{2}}{V}
$$

where $\rho_{E c}$ is the energy density of space in the centre of a black hole, $\mathrm{m}$ is the mass of the black hole and $V$ is its volume.

\section{Calculation of the "Schwarzschild energy density"}

"Schwarzschild energy density" one can calculate using Eq. (2):

$$
\rho_{E . S c h .}=\rho_{E P}-\frac{3 m_{\odot} c^{2}}{4 \pi r_{S c h}^{3}}
$$

where $m_{\odot}$ is the mass of the Sun, and its correspondent Schwarzschild radius $r_{\text {Sch. }}$ is $3 \cdot 10^{3} \mathrm{~m}$.

$$
\rho_{\text {E.Sch. }}=4.64 \cdot 10^{113} \mathrm{Jm}^{-3}-1.58 \cdot 10^{35} \mathrm{Jm}^{-3} \text {. }
$$

When in the centre of the stellar object the value of energy density of space $\rho_{E c}$ is smaller as Schwarzschild energy density, the atoms in the centre become unstable and are falling apart into elementary particles:

$$
\rho_{E c}<\rho_{E . S c h .} \rightarrow \text { atoms are unstable }
$$

The Schwarzschild energy density offers a new interpretation of space-time singularities in the centre of a black hole: "If, as seems justifiable, actual physical singularities in space-time are not to be permitted to occur, the conclusion would appear inescapable that inside such a collapsing object at least one of the following holds: (a) Negative local energy occurs. (b) Einstein's equations are violated. (c) The space-time manifold is incomplete. (d) The concept of space-time loses its meaning at very high curvature - possible because of quantum phenomena. In fact (a), (b), (c), (d) are somewhat interrelated, the distinction being partly one of attitude of mind" [6]. I suggest that space-time singularity in the centre of black hole indicate that in the centre of a black hole exist critical physical circumstances that I previously defined as "the energy density of space is below the Schwarzschild energy density $\rho_{E . S c h}$.".

According to Newton's Shell theorem in space-time singularity, gravity force does not tend to the infinite value; it tends to zero. Going inside the black hole at the distance $d$ from the surface 
towards the centre gravity force on a given object with mass $m$ is diminishing regarding the gravity force on the surface according to the Eq. (3):

$$
F_{g 1}=\frac{m M_{1} G}{r_{1}}
$$

where $m$ is the mass of a given object, $M_{1}$ is the mass of the black hole shell with the radius $r_{1}$, and $G$ is gravitational constant. When $r_{1}$ is tending to the zero, $M_{1}$ is also tending to the zero, and gravity force $F_{g 1}$ is also tending to the zero:

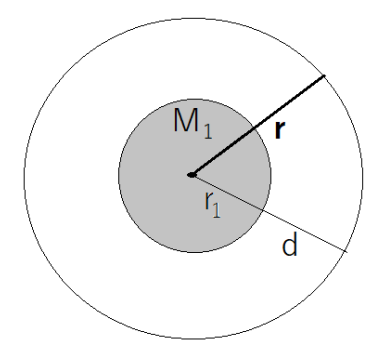

Figure1: Gravity force inside black hole

In the centre of the black hole, there is no gravity force. The extreme physical circumstance in the centre of the black hole is that the energy density of space there is below Schwarzschild energy density. This model is adding to the understanding of the interior of black holes which back in 2000 was still an open question: "We have confined ourselves in this paper to a specific example that relaxes the condition of asymptotic flatness while preserving time-symmetry. The starting point here is the static black hole in the Einstein universe which belongs to the family of solutions presented by Vaidya. In this spacetime the black hole is well defined as the Killing horizon. However, the nature of the interior of the black hole is not entirely clear" [7].

\section{Variable energy density of space at the distance $d$ from the centre of the stellar objects}

The energy density of the universal space $\rho_{E d}$ at the distance $d$ from the centre of a given stellar object with mass $m$ and radius $r$ is calculated using the equation below that is developed on the basis of the Eq. (2):

$$
\rho_{E d}=\rho_{E P}-\frac{3 m c^{2}}{4 \pi(r+d)^{3}}
$$

When $d$ is zero, Eq. (5) is equal to the Eq. (2), when $d$ tents towards the infinite, energy density $\rho_{E d}$ tends towards Planck energy density $\rho_{P}$. We will use this formula to calculate the energy 
density of space in the centre of different stellar objects, considering that these objects are nonrotating. In Table 1 there is the comparation of the energy densities of space in the centre of the black hole with the mass of the Sun, in the centre of the proton, in the centre of the Moon, Earth, and Sun:

Table 1. Comparation values of the minimal energy density of space with respect to the centre of indicated objects.

\begin{tabular}{|c|c|}
\hline Centre of objects & $\rho_{P}=4.64 \cdot 10^{113} \mathrm{Jm}^{-3}$ \\
\hline Black hole with mass of the Sun & $\rho_{P}-1.58 \cdot 10^{36} \mathrm{Jm}^{-3}$ \\
\hline Proton & $\rho_{P}-5.43 \cdot 10^{34} \mathrm{Jm}^{-3}$ \\
\hline Earth & $\rho_{P}-4.94 \cdot 10^{20} \mathrm{Jm}^{-3}$ \\
\hline Moon & $\rho_{P}-3.00 \cdot 10^{20} \mathrm{Jm}^{-3}$ \\
\hline Sun & $\rho_{P}-1.26 \cdot 10^{20} \mathrm{Jm}^{-3}$ \\
\hline
\end{tabular}

In the centre of a proton, the minimal energy density of space is for the order of $10^{2}$ too high for the proton to become a mini black hole as proposed by Hawking [8]. Voyager did not discover mini black holes in interstellar space [9]. The energy density of space in the proton centre is lower than in the centre of Sun, Earth and Moon because these stellar objects are made out of atoms where there is a vast empty space between the nucleus and electrons orbits. Proton's mass is very small compared with the mass of the Sun, but it diminishes the energy density of an extremely small area of space compared with that of Sun, that diminishes the energy density of an extremely big area of universal space; that's why the gravity force of the Sun has such a long-range.

We will compare the energy density of space of a stationary black hole with the mass of the Sun and energy density of space of the Sun at given distances from the centre (Table 2). 
Table 2. Comparation values of the minimal energy density of space with respect to the distance by centre of indicated objects.

\begin{tabular}{|c|c|c|c|}
\hline Sun centre & $\rho_{P}-1.26 \cdot 10^{20} \mathrm{Jm}^{-3}$ & Black hole centre & $\rho_{P}-1.58 \cdot 10^{36} \mathrm{Jm}^{-3}$ \\
\hline \multicolumn{2}{|c|}{ Distance from the centre } & \multicolumn{2}{|c|}{ Distance from the centre } \\
\hline $10^{2} \mathrm{~km}$ & $\rho_{P}-1.26 \cdot 10^{20} \mathrm{Jm}^{-3}$ & $10^{2} \mathrm{~km}$ & $\rho_{P}-3.90 \cdot 10^{31} \mathrm{Jm}^{-3}$ \\
\hline $10^{3} \mathrm{~km}$ & $\rho_{P}-1.26 \cdot 10^{20} \mathrm{Jm}^{-3}$ & $10^{3} \mathrm{~km}$ & $\rho_{P}-4.22 \cdot 10^{28} \mathrm{Jm}^{-3}$ \\
\hline $10^{4} \mathrm{~km}$ & $\rho_{P}-1.21 \cdot 10^{20} \mathrm{Jm}^{-3}$ & $10^{4} \mathrm{~km}$ & $\rho_{P}-4.25 \cdot 10^{25} \mathrm{Jm}^{-3}$ \\
\hline $10^{5} \mathrm{~km}$ & $\rho_{P}-8.44 \cdot 10^{19} \mathrm{Jm}^{-3}$ & $10^{5} \mathrm{~km}$ & $\rho_{P}-4.25 \cdot 10^{19} \mathrm{Jm}^{-3}$ \\
\hline $10^{6} \mathrm{~km}$ & $\rho_{P}-8.73 \cdot 10^{18} \mathrm{Jm}^{-3}$ & $10^{6} \mathrm{~km}$ & $\rho_{P}-4.26 \cdot 10^{19} \mathrm{Jm}^{-3}$ \\
\hline $0.01 \mathrm{AU}$ & $\rho_{P}-2,00 \cdot 10^{14} J m^{-3}$ & $0.01 \mathrm{AU}$ & $\rho_{P}-2.07 \cdot 10^{14} J^{-3}$ \\
\hline $0.1 \mathrm{AU}$ & $\rho_{P}-2.07 \cdot 10^{11} \mathrm{Jm}^{-3}$ & $0.1 \mathrm{AU}$ & $\rho_{P}-2.07 \cdot 10^{11} \mathrm{Jm}^{-3}$ \\
\hline $0.5 \mathrm{AU}$ & $\rho_{P}-1.66 \cdot 10^{9} \mathrm{Jm}^{-3}$ & $0.5 \mathrm{AU}$ & $\rho_{P}-1.66 \cdot 10^{9} \mathrm{Jm}^{-3}$ \\
\hline $1 \mathrm{AU}$ & $\rho_{P}-2,07 \cdot 10^{8} \mathrm{Jm}^{-3}$ & $1 \mathrm{AU}$ & $\rho_{P}-2.07 \cdot 10^{8} \mathrm{Jm}^{-3}$ \\
\hline
\end{tabular}

Going from the centre of the black hole, the energy density of the space is increasing at a much higher rate than going away from the centre of the Sun. At the distance of $0.01 \mathrm{AU}$ from the centre of both stellar objects, the energy density of space is at the same rate of $-2 \cdot 10^{14} \mathrm{Jm}^{-3}$ comparing the Planck energy density and is increasing by the same values with the increase of the distance.

\section{The quantum mechanism of AGNs' jets}

In the centre of black holes, atoms are transforming back into elementary particles. This creates enormous pressure and if gravity pressure of the black hole is not big enough, such a black hole explodes in a supernova [10]. When the black hole gravity pressure is strong enough, as it is the case for example with the black hole in the quasar SMSSJ215728.21-360215.1 which has about $(3.4 \pm 0.6) \cdot 10^{10} M_{\odot}[11]$, the transformation of matter into elementary particles creates the explosion that opens the hole in the direction of the rotational axis (Figure 2). 


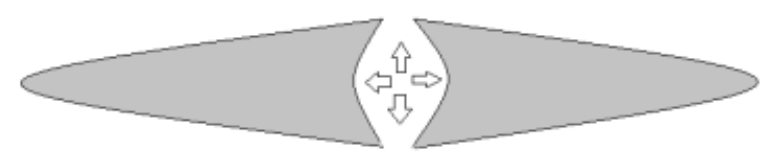

Figure 2: Cross-section of a black hole in the centre of the quasar SMSSJ215728.21-360215.1

Through this hole, in the direction of rotation, the black hole is throwing a jet of elementary particles into the intergalactic space (Figure 2).

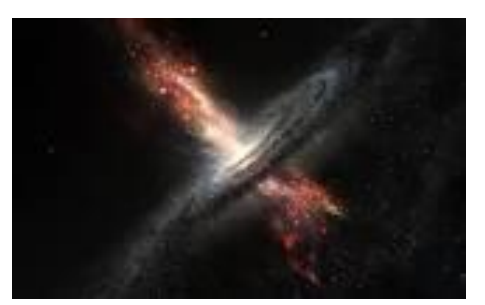

Figure 3: Jets of a black hole in the centre of a galaxy (with permission of Southern European observatory).

Centres of AGN's where energy density of space is lower than Sch. energy density are a kind of quantum mechanisms where the matter falls apart into elementary particles and forms jests. We give in this article a solution to the mystery of jets production following Einstein's idea that matter can be transformed into energy and vice versa: "Relativistic magnetized jets from active galaxies are among the most powerful cosmic accelerators, but their particle acceleration mechanisms remain a mystery" [12]. These jets are building material for new stars formation; black holes are then rejuvenating systems of the universe: "old" matter is transformed into "fresh" energy in the form of AGNs jets.

\section{Conclusions}

The law of energy conservation requires that AGN's jets must have some physical sources. It is shown in this article that these jets are originated in the process of matter falling apart in the centres of AGNs, where there are space-time singularities and energy density of space is below the Schwarzschild energy density. 


\section{References:}

1. Sbitnev, V.I. Hydrodynamics of the Physical Vacuum: II. Vorticity Dynamics. Found Phys 46, 1238-1252 (2016). https://doi.org/10.1007/s10701015-9985-3.

2. Sbitnev, V.I. Hydrodynamics of the Physical Vacuum: I. Scalar Quantum Sector. Found Phys 46, 606-619 (2016). https://doi.org/10.1007/s10701-0159980-8.

3. 3. Fiscaletti, D., \& Sorli, A.S. Quantum Relativity: Variable Energy Density of Quantum Vacuum as the Origin of Mass, Gravity and the Quantum Behaviour. Ukr. J. Phys. 63(7), 623 (2018). https://doi.org/10.15407/ujpe63.7.623.

4. Šorli, A.S. Mass-Energy Equivalence Extension onto a Superfluid Quantum Vacuum. Sci Rep 9, 11737 (2019). https://doi.org/10.1038/s41598-019-48018-2.

5. Misner, C.W., Thorne, K.S. Wheeler, J.A. Gravitation. San Francisco: W.H. Freeman, 875-876 (1973).

6. Penrose, R. Gravitational Collapse and Space-Time Singularities. Phys. Rev. Lett. 14, 57 (1965). https://doi.org/10.1103/PhysRevLett.14.57.

7. Nayak, K.R., Mac Callum, M.A.H., and Vishveshwara, C.V. Black Holes in Nonflat Backgrounds: the Schwarzschild Black Hole in the Einstein Universe (2000). https://arxiv.org/pdf/gr-qc/0006040v1.pdf.

8. Hawking, S. W. Gravitationally collapsed objects of very low mass. MNRAS $\mathbf{1 5 2}$, 7 (1971). https://doi.org/10.1093/mnras/152.1.75.

9. Boudaud, M. \& Cirelli, M. Voyager 1 e \pm Further Constrain Primordial Black Holes as Dark Matter. Phys. Rev. Lett. 122, 041104 (2019). https://doi.org/10.1103/PhysRevLett.122.041104.

10. Croswell K., Inner Workings: A massive star dies without a bang, revealing the sensitive nature of supernovae, PNAS January 21, 2020117 (3) 12401242; https://doi.org/10.1073/pnas.1920319116.

11. Onken, C.A., Bian, F., Fan, X., Wang, F., Wolf, C., Yang, J. A thirty-four billion solar mass black hole in SMSS J2157-3602, the most luminous known quasar. MNRAS 496(2), 2309-2314 (2020). https://doi.org/10.1093/mnras/staa1635.

12. Alves E.P., Zrake, J., Fiuza, F. Efficient Nonthermal Particle Acceleration by the Kink Instability in Relativistic Jets, Phys. Rev. Lett. 121, 245101 (2018). https://arxiv.org/abs/1810.05154. 
